are based in part on measurements of the state of development of the gut microbial community.

Although that envisioned approach for evaluating MDCFs brings a microbial and 'multi-omics' perspective to human-nutrition research, it presents a variety of challenges for organizations such as the WHO and UNICEF that formulate recommendations for treating malnourished children. These challenges include balancing a desire to aggregate children over broad age ranges (e.g., from 6 months to 24 or even 36 months) on the basis of their anthropometric scores with the inevitably more stratified view of human biology that is revealed when co-development of the microbiome and host is considered; how this balancing act is negotiated could have substantial therapeutic and policy implications ${ }^{11}$. A list of other considerations for MDCF development that are related to science and technology, social and cultural considerations, and policy and governance is provided (Table 1).

Research and development in this area needs to be incentivized in ways that ensure equitable and widespread distribution of the fruits of such efforts. A promising system developed by Nutriset, the largest manufacturer of ready-to-use foods for malnourished children globally, relies on a franchise model that provides local stakeholders the intellectual property, tools and expertise to produce its therapeutic foods $s^{5}$. Such a model leverages patent protection to create financial incentives for local manufacturers by effectively making intellectual property inaccessible to large manufacturing companies in high-income countries that could potentially monopolize production. Although focusing on local production may sacrifice short-term economies of scale for sustainability, a campaign to develop and deploy MDCFs must include long-term strategies for developing nations to achieve nutritional autonomy ${ }^{12}$.

Looking ahead, it is important to determine whether microbiome-directed therapeutic foods can be developed for women of childbearing age to maximize their nutritional health, minimize low birth weight in their offspring and help break the vicious cycle of intergenerational malnutrition. Additionally, can knowledge gained from identifying foods that repair the microbiome of children with malnutrition also be used to identify a temporal sequence of complementary foods that prevent failed microbial community development and thus promote healthy growth? These questions lie at the base of a new kind of food pyramid that considers the gut microbiome as part of nutrition itself.

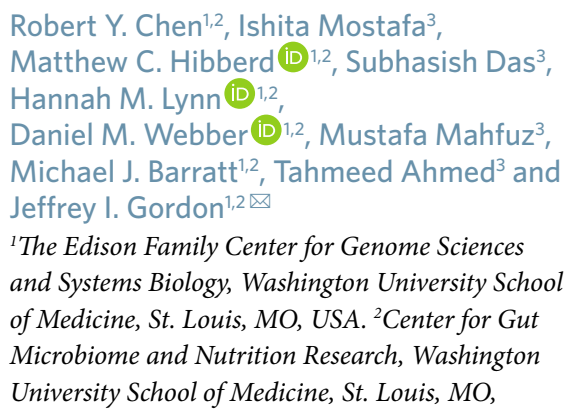

USA. International Centre for Diarrhoeal Disease Research, Bangladesh, Dhaka, Bangladesh.

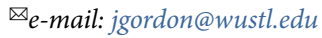

Published online: 29 July 2021

https://doi.org/10.1038/s41591-021-01451-1

References

1. UN General Assembly. https://www.un.org/en/development/desa/ population/migration/generalassembly/docs/globalcompact/A RES_70_1_E.pdf (2015).

2. UNICEF. https://data.unicef.org/resources/ state-of-the-worlds-children-2019/ (2019).

3. WHO Multicentre Growth Reference Study Group. Acta Paediatr. 450, 76-85 (2006).

4. Raman, A. S. et al. Science 365, eaau4735 (2019).

5. Gehrig, J. L. et al. Science 365, eaau4732 (2019).

6. Chen, R. et al. N. Engl. J. Med. 384, 1517-1528 (2021).

7. Kolodziejczyk, A. A., Zheng, D. \& Elinav, E. Nat. Rev. Microbiol. 17, 742-753 (2019).

8. Amicucci, M. J. et al. Nat. Commun. 11, 1-12 (2020).

9. Dmitrienko, A. \& D’Agostino, R. B. Sr. N. Engl. J. Med. 378, 2115-2122 (2018)

10. Wesener, D. A. et al. eLife 10, e64478 (2021)

11. Wilkinson, J. E. et al. Nat. Med. 27, 766-774 (2021).

12. Sanderson, J. in The Intellectual Property and Food Project 145-173 (Routledge, 2014).

\section{Acknowledgements}

Work performed in the authors' labs described in this Comment was supported by the Bill \& Melinda Gates Foundation and the US National Institutes of Health. We are indebted to our fellow investigators of the Breast Milk, Gut Microbiome and Immunity Project, including N.N. Naila, M.M. Islam, S. Huq, M.A. Alam, M.U. Zaman, H.-W. Chang, Y. Wang, A. Osterman, D. Rodionov, C. Newgard and C. Lebrilla.

\section{Author contributions}

The Comment was written by R.Y.C. and J.I.G. with input from all co-authors.

\section{Competing interests}

The authors declare no competing interests.

\title{
Malaria: a problem to be solved and a time to be bold
}

\author{
Vaccines alone will not be sufficient for the eradication of malaria, which will also require investment in health \\ professionals, better use of data, and universal access to quality health services.
}

\section{Pedro L. Alonso}

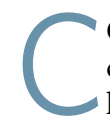

OVID-19 has provided a wake-up call that infectious diseases can have huge health, economic and social costs and require investments that protect the wellbeing of people across the world. By threatening the health and economies of all countries, unprecedented financial efforts and incentives were deployed in a very short period of time to develop and implement new tools against COVID-19, especially vaccines.
This unprecedented investment has revealed the extraordinary power of science, delivering several safe and effective vaccines within months of the sequence of SARS-CoV-2 being determined and shared. 


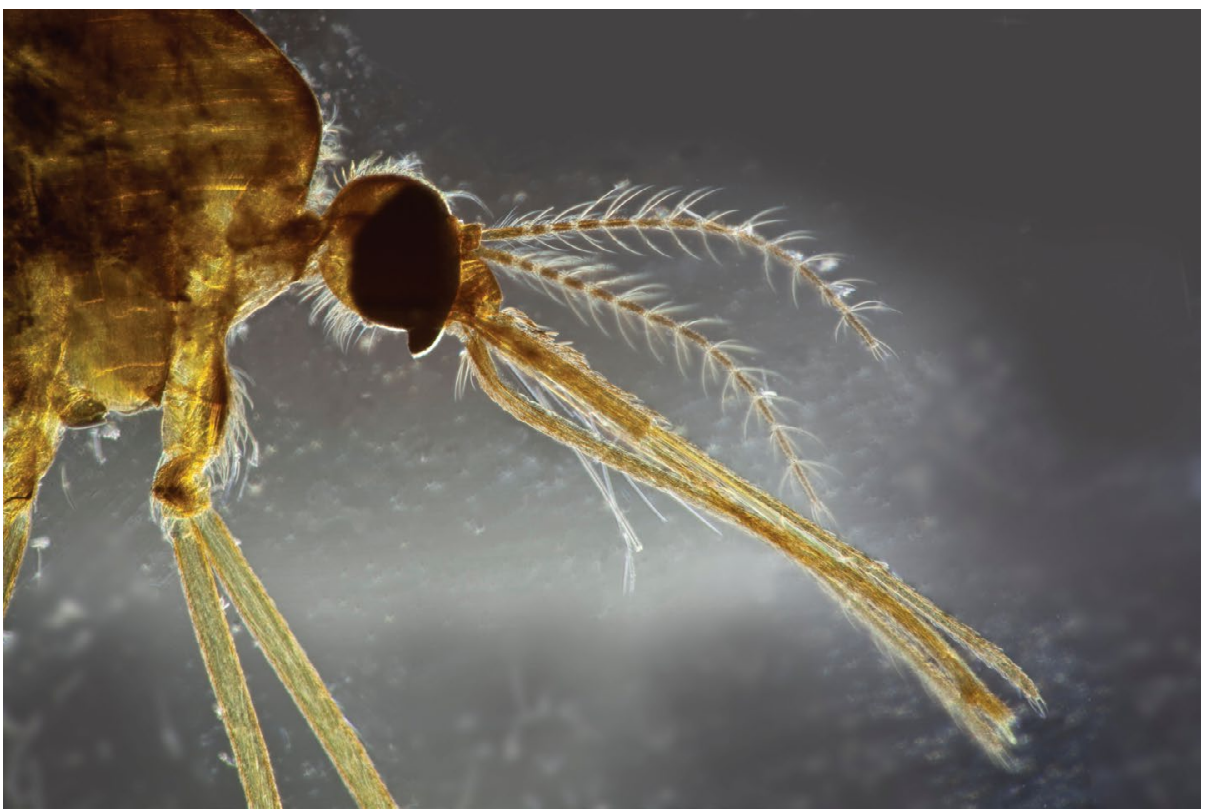

Photomicrograph of Anopheles sp. mosquito, showing the head and mouthparts. Credit: Scenics \& Science/Alamy Stock Photo

The investment and benefits from science, coupled with global solidarity and a focus on equity, should ensure sufficient supply and the equitable distribution of vaccines. It should also be extended to other diseases, such as malaria, that currently threaten the lives of millions of people around the world.

\section{A golden era}

History demonstrates that such an investment in malaria can reap remarkable benefits. At the start of the twenty-first century, the transmission of malaria was taking place in 107 countries across five continents, where more than $80 \%$ of the world's population lived ${ }^{1}$. Sub-Saharan Africa carried the brunt of the disease, where a child probably died of malaria every 45 seconds and efforts to control the disease were very limited. Yet this desperate situation had not gone unnoticed.

Towards the end of the last century, there was a growing political and scientific momentum that would lead to a golden era in the fight against this ancient killer of humankind. Insecticide-treated bed nets, artemisinin-based combination therapies, rapid diagnostic tests, new approaches to chemoprevention of target groups with drugs (such as intermittent preventive treatment in pregnancy or in infants, and seasonal malaria chemoprevention) were the extraordinary outputs of a small and underfunded research community.

On the political front, the Abuja Declaration by African Heads of State, followed by the inclusion of malaria in the
United Nations Millennium Development Goals, was accompanied by the creation of new funding mechanisms such as The Global Fund to Fight AIDS, Tuberculosis and Malaria, and the US President's Malaria Initiative $^{2-5}$. For the first time, substantial financial resources ensured that antimalarial tools could be procured and delivered at scale. The effect has been staggering: 7.6 million deaths and 1.5 billion cases averted in the first 20 years of this century ${ }^{1}$.

These gains have also occurred at a time when many malaria endemic countries have experienced considerable economic growth and social development, both of which are important contributors to decreasing malaria burden. In sub-Saharan Africa, GDP has grown by an average of $4 \%$ per annum in the period from 2000 to $2019^{6}$. During the same time, access to electricity has increased threefold ${ }^{7}$ and the percentage of the African population in urban settings has increased from $31 \%$ to $41 \%$ (ref. ${ }^{8}$ ). It is the combined efforts from the scientific community, leadership across the world and socioeconomic development that have contributed to a public health success story and a great return on global health investment.

\section{Two tiers}

However, the past five years have shown both the success and the limitations of this effort ${ }^{1}$. More than half (46) of the 87 malaria endemic countries in 2019 are within reach of eliminating the transmission of malaria within their borders. These countries, mostly outside Africa, now account for less than $0.2 \%$ of all malaria cases globally, and some, such as El Salvador and China, have recently been certified malaria free ${ }^{1,9,10}$.

However, for a considerable proportion of the remaining countries with ongoing malaria transmission, reductions in disease incidence and mortality rates have slowed down, particularly in sub-Saharan Africa, where $94 \%$ of global malaria cases and deaths occur.

Funding, from both international partners and the endemic countries themselves, has stalled. In the face of a doubling of the population over the past 20 years in sub-Saharan Africa alone, the stark reality is that after US $\$ 26$ billion of investment to tackle malaria in this region, the estimated number of malaria cases are slightly higher in 2019 (215 million) than in 2000 (204 million $)^{1}$.

The world is therefore likely to continue to see success as a good number of countries become malaria free. However, we are not on track to achieve the agreed targets for reductions in morbidity and mortality by 2030 as set out in the WHO Global Technical Strategy ${ }^{11}$, and malaria eradication is not within sight. In 2017, the WHO raised the alarm on the stalling of progress by declaring that the world was at a crossroads in the fight against malaria ${ }^{12,13}$.

\section{Sustaining the gains}

First, more financial resources are required. Financing mechanisms and governance also need to acknowledge and enable the leadership of malaria endemic countries, who in turn need to take greater financial responsibility.

Second, plans and activities to control malaria have to be imbedded in the Universal Health Coverage and Primary Health Care agenda ${ }^{14,15}$. Robust, resilient, quality health systems are essential to tackle malaria.

Third, data can also support subnational operations, inspired by a problem-solving mindset, to move away from a one-size fits all approach. Countries must invest in quality health-management information systems. The scale up of point-of-care malaria diagnosis, the gradual switch to electronic databases and the investment in malariometric surveys in recent years, which determine the level of malaria in specific locations, have led to increasing availability of reasonable quality data ${ }^{1}$.

Geospatial and mathematical modeling approaches can take into account the inherent heterogeneity of malaria transmission as well as contextual elements, such as access to health facilities, urbanization, important social determinants 
and other factors. This allows stratification at a national and subnational level, empowering governments to reliably define the optimal mix of malaria interventions that will achieve maximum effect within a given resource envelope.

This sub-nationally tailored approach must extend beyond what is delivered to include local decisions of how to efficiently and equitably deliver to those in need. A sub-national approach will also require further investments in improving surveillance systems, establishment of dynamic integrated national repositories to systematically curate relevant data and national capacity to analyze and make use of the data for locally tailored responses.

Fourth, the malaria community needs to acknowledge the strength and limitations of the tools and strategies available today. We should be able to diagnose and treat all malaria cases. Affordable and easy to use point-of-care diagnostics allow a parasitological confirmatory test. Similarly, safe and highly effective antimalarial medicines exist and can be used to treat all infections. Consequently, no one should be dying of malaria. However, more than 400,000 malaria deaths continue to take place every year owing to a lack of access to prompt quality care.

\section{Preventable deaths}

Prevention relies on both tools against the anopheline vector, such as insecticide-treated mosquito nets, and the use of medicines to prevent infections in key target groups. When properly implemented, prevention with medicine results in a substantial reduction in disease and malaria deaths.

However, a lack of prioritization in research and development means that there is a lack of optimal drugs, regimes and formulations for prevention, which represent a barrier to adoption and impact. Furthermore, both drugs and diagnostics will remain challenged by the emergence and spread of drug resistance or parasite gene deletions, a threat towards which the malaria community has so far responded effectively.

In most of sub-Saharan Africa, long-lasting insecticide-treated nets (LLINs) represent the cornerstone of efforts to control the malaria vector. The efficacy of LLINs in the prevention of disease is modest-around $45 \%$ in children under the age of five ${ }^{16}$ - and the nets need to be replaced every three years. Part of the efficacy of LLINs relies on impregnation with insecticide, against which Anopheline mosquitoes can develop resistance, and they also rely on quality and integrity, which may represent an even greater challenge ${ }^{17}$.
New prevention tools are needed, including against the mosquito vector and antimalarial medicines. Monoclonal antibodies may also provide opportunities to prevent infection for several months during the periods of highest risk for the key target populations.

\section{Various vaccines}

COVID-19 reminds us that vaccines remain the most important tool to prevent communicable diseases. The quest for a malaria vaccine started more than a century ago. Today, there is a first-generation malaria vaccine (RTS,S produced by GSK) based on a recombinant protein that targets the circumsporozoite protein, the predominant sporozoite surface protein of Plasmodium falciparum. RTS,S has completed its clinical development and received a positive scientific opinion by the European Medicines Agency ${ }^{18}$. The efficacy of RTS,S is modest - around $40 \%$ reduction of disease.

RTS,S is currently undergoing large-scale pilot implementation that involves several hundreds of thousands of children in three African countries, and will be considered for a potential WHO recommendation for broader scale use before the end of the year. If this historic decision is reached, it would represent the first time that a vaccine against a human malaria parasite is recommended for public-heath use and a first vaccine incorporated to the antimalarial armamentarium toolbox, with the potential to avert millions of cases and hundreds of thousands of deaths. More importantly, this first-generation vaccine will show that the 30-year-long development effort has yielded a safe and effective vaccine against a complex parasite.

A limited number of other vaccine candidates are being tested. One of the most advanced is the $\mathrm{R} 21 / \mathrm{MM}$ vaccine candidate. This is based on a similar circumsporozoite antigen to RTS,S and has been developed using the Matrix-M adjuvant platform. In a phase $2 \mathrm{~b}$ trial, $\mathrm{R} 21 / \mathrm{MM}$ showed $77 \%$ protection over a one-year follow up, among 450 children living in an area of intensely seasonal malaria transmission in Burkina Faso $^{19}$. The results of the phase $2 \mathrm{~b}$ trial are encouraging, and has increased anticipation for phase 3 trials that, crucially, need to include areas where malaria occurs all year round, as well as long-term follow up to allow adequate comparison with RTS,S.

In recent years, the use of whole parasite immunization strategies has been tried. Whole-cell P. falciparum sporozoite (PfSPZ)-based vaccines are a promising way to evoke immunity, as a broad antigenic repertoire of the parasite is present in the pre-erythrocytic development stages, especially in the liver phase. Sporozoites either attenuated by radiation, or with their development arrested via antimalarial medicines, have been reported to induce $100 \%$ protection against heterologous challenge in a small number of volunteers ${ }^{20}$.

BioNTech has recently announced its intention to develop a malaria vaccine using its mRNA technology, which has been used so effectively against COVID-19 ${ }^{21}$. The goal is to yield a tool that will enable eradication, with a high efficacy in preventing infection and with a long duration of protection. This declaration represents a U-turn in the decades-long trend of major vaccine developers abandoning or scaling down their efforts in the field of malaria. This announcement also represents the potential of new visions and approaches from the field of oncology being applied to address the challenges and complexities of inducing immunity against complex parasites.

\section{Be bold}

Malaria has plagued humans for millennia and has led to an unimaginable loss of life. Malaria has also had an important role in the geopolitics and evolutionary history of humans. The malaria problem is evolving, dynamic and diverse, but it is now concentrated in some of the poorest communities in the world. The lessons of the past two decades show that success in malaria is possible when the world pulls together. However, enormous biological, political, governance, socioeconomic, data and financial challenges remain. These challenges require the world to be bold again.

New tools, including vaccines, could represent game changers, and demand a collective effort that builds on scientific learning and collaboration. But vaccines by themselves will not be a solution to the problem of malaria. No progress will be made without a well-trained and empowered cadre of health workers. Better use of data to plan, implement and track progress, even within a single country, will allow resources to be used more efficiently.

Finally, national decision-making processes must be at the core of public health governance. Governments must identify and serve those communities that are consistently not reached with quality malaria services, especially those delivered through the public health system. Only then can we move again toward global eradication.

Pedro L. Alonso

Global Malaria Programme, World Health

Organization, Geneva, Switzerland.

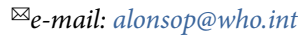


Published online: 13 September 2021

https://doi.org/10.1038/s41591-021-01492-6

References

1. World Health Organization. https://www.who.int/publications/i/ item/9789240015791 (2020).

2. World Health Organization. https://apps.who.int/iris/handle/ 10665/67816 (2000)

3. United Nations. https://www.un.org/millenniumgoals/ (2015).

4. The Global Fund. https://www.theglobalfund.org/en/overview/ (2019).

5. PMI. https://www.pmi.gov/about (2015).

6. The World Bank. https://data.worldbank.org/indicator/NY.GDP PCAP.CD (2020).

7. The World Bank. https://data.worldbank.org/indicator/EG.ELC ACCS.RU.ZS (2020)

8. United Nations. https://population.un.org/wpp/ (2019).
9. PAHO. https://www.paho.org/en/topics/malaria/salvadorfirst-central-american-country-receive-who-malaria-free-country (2021).

10. World Health Organization. https://www.who.int/news/item/ 30-06-2021-from-30-million-cases-to-zero-china-is-certified malaria-free-by-who (2021)

11. World Health Organization. https://www.who.int/malaria/areas/ global_technical_strategy/en/ (2015).

12. World Health Organization. https://www.who.int/malaria/ publications/world-malaria-report-2017/en/ (2017)

13. Alonso, P. \& Noor, A. M. Lancet 390, 2532-2534 (2017).

14. World Health Organization. https://www.who.int/teams/ primary-health-care/conference/declaration (2018)

15. United Nations. https://www.un.org/pga/73/event/ universal-health-coverage/ (2019)

16. Lengeler, C. Cochrane Database Syst. Rev. 2, CD000363 (2004)

17. Lindsay, S. W., Thomas, M. B. \& Kleinschmidt, I. Lancet 9, e1325-e1331 (2016)
18. World Health Organization. https://www.who.int/immunization/ sage/meetings/2015/october/1_Final_malaria_vaccine_ background_paper_v2015_09_30.pdf (2015).

9. Datoo, M. S. et al. Lancet 397, 1809-1818 (2021).

20. Mwakingwe-Omari, A. et al. Nature 595, 289-294 (2021).

21. Cheney, C. https://www.devex.com/news/plans-for-new-malariavaccine-underscore-benefits-of-mrna-beyond-covid-19-100497 (2021).

Acknowledgements

This manuscript has benefitted from several discussions with partners and colleagues within the Global Malaria Programme, particularly A. Noor, D. Schellenberg and A. Robb.

Competing interests

The author declares no competing interests. 